\title{
A massive pericardial effusion in South Africa is not always due to tuberculosis
}

Sipho Ntshalintshali, Nontembiso Mhlana, Farzana Moosajee, Riyad Abousriwiel, Kiran George and Riette du Toit

Division of Rheumatology, Department of Internal Medicine, Stellenbosch University and Tygerberg Hospital, Bellville, South Africa

Address for correspondence:

Dr Sipho Ntshalintshali

Division of Rheumatology

Department of Internal Medicine

Stellenbosch University and Tygerberg Hospital

Francie van Zijl Avenue

Bellville

7505

South Africa

\section{Email:}

sipho.duncan@gmail.com

\section{INTRODUCTION}

Massive pericardial effusions can be due to multiple etiologies. In South Africa, tuberculosis is a common cause of massive pericardial effusion. sJIA and AOSD, which usually present in $5 \%$ - $10 \%$ of patients as pyrexia or fever of unknown origin (FUO) accompanied by systemic manifestations, can be associated with a massive pericardial effusion and mimic tuberculosis.

\section{CASE REPORTS}

We report on a 15-year-old male, HIV uninfected, who presented with a 3-week history of a productive cough, dyspnoea, fever, weight loss and multiple large joint arthralgia. On examination he was tachycardic, normotensive, had a respiratory rate of 22 breaths per minute, and had a fever with a quotidian pattern (Figure I). He had cervical, axillary and inguinal lymphadenopathy of approximately $1 \mathrm{~cm}$. On cardiovascular and respiratory examination he had a small volume and tachycardic pulse, with distended neck veins, audible normal heart sounds with no pericardial rub, and bilateral coarse crackles. He had inflammatory polyarthritis involving the wrists, elbows, knees, and ankles. No skin rash was detected and other organ system findings were unremarkable.

\section{ABSTRACT}

South Africa (SA) has a high incidence of tuberculosis. Medical conditions mimicking tuberculosis often result in erroneous treatment with antitubercular therapy (ATT) before a definitive diagnosis is made. We report on 2 cases presenting with massive pericardial effusions secondary to Still's disease (sJIA) and Adult onset Still's disease (AOSD). Both cases were treated with ATT, with an alternative diagnosis only considered upon poor response to therapy and the development of ATTassociated side effects. Our objective is to remind clinicians of other potential differential diagnoses in the clinical scenario of massive effusive pericarditis in a tuberculosis endemic region. SAHeart 2020; 17:204-207

Radiographs of involved joints were normal while the chest radiograph showed an increased cardiothoracic ratio (CTR), while the echocardiogram revealed a massive pericardial effusion, and pericardiocentesis was done. The abdominal ultrasonography was normal. The blood test results are summarised in Table I. A decision to start empiric ATT was made considering the local high incidence of tuberculosis. A week later he had no clinical improvement but developed a druginduced liver injury and ATT was discontinued. SJIA was considered as a more likely alternative diagnosis since the patient fulfilled the Yamaguchi criteria, and was given a 750mg intravenous pulse of solumedrol daily over a 3-day period, followed by a remarkable clinical response. Within 2 weeks he was discharged on prednisone at $0.5 \mathrm{mg} / \mathrm{kg} /$ day and azathioprine $50 \mathrm{mg}$ daily as a steroid sparing agent.

The second case is that of a 28-year-old male with a background of dengue fever 3 years prior, who now presented with a I-week history of a pharyngitis, polyarthralgia, and chest pain with shortness of breath. He presented with Beck's triad for a pericardial tamponade with a blood pressure of 65/45, heart rate of 130 beats per minute, and a respiratory rate of 28 breaths per minute. He had a quotidian pattern fever, a non-palpable, non-blanching vasculitic type rash on both shins, and small cervical lymphadenopathy. He also had bilateral small knee effusions. Other organ-system findings were unremarkable.

The electrocardiogram showed a sinus tachycardia with microvoltage. The chest $X$-ray revealed an increased CTR (Figure 2), and on echocardiogram had evidence of a cardiac tamponade 


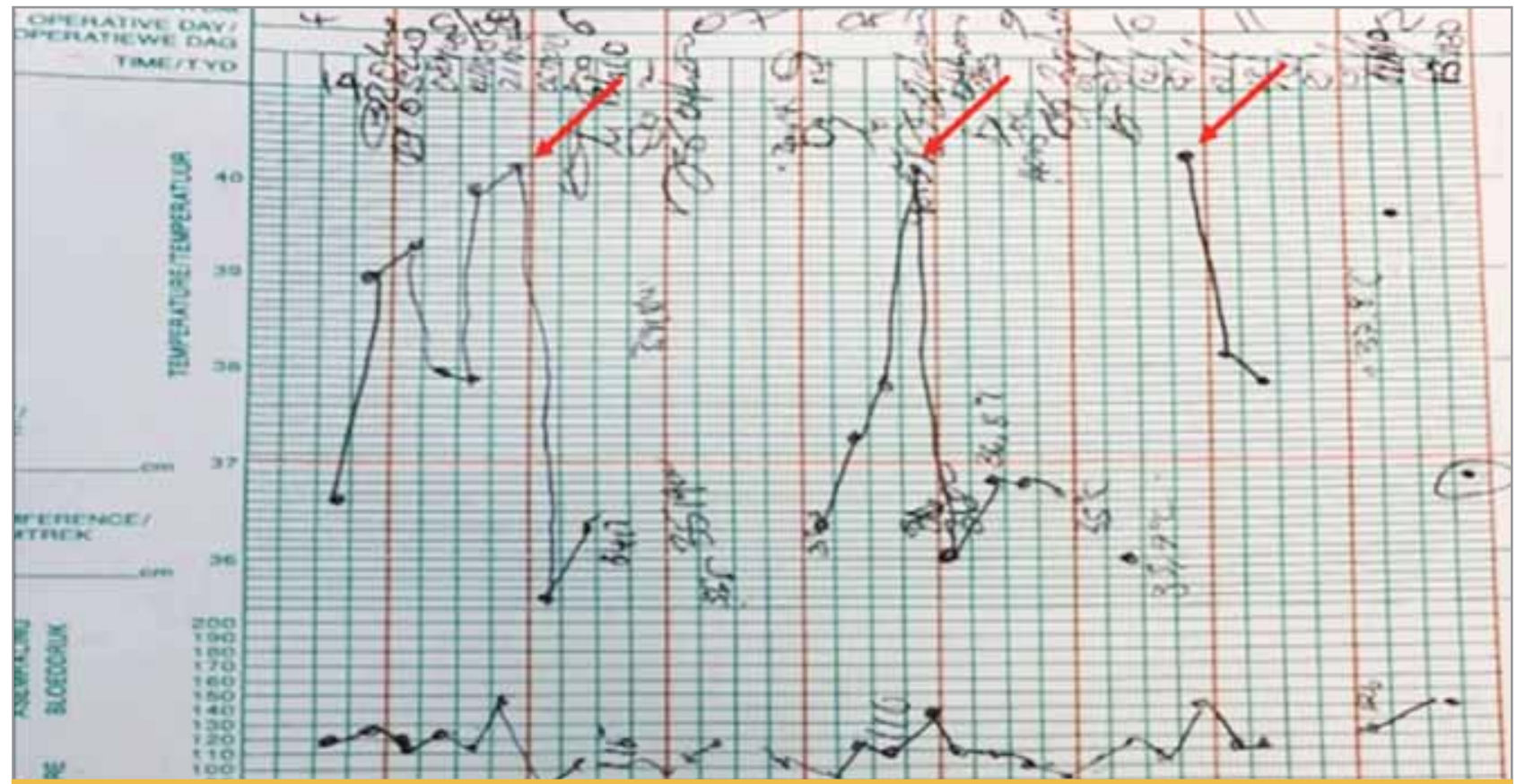

FIGURE I: Red arrows: Daily fever spikes of up to $40^{\circ} \mathrm{C}$ (quotidian pattern).

TABLE I: Laboratory investigations on admission.

\begin{tabular}{|c|c|c|}
\hline Investigation & Case I & Case 2 \\
\hline WCC $\left(\times 10^{9} / \mathrm{L}\right)$ & 51 & 30.36 \\
\hline $\mathrm{Hb}(\mathrm{g} / \mathrm{dL})$ & 8.6 & 8.7 \\
\hline Plt $\left(\times 10^{9} / \mathrm{L}\right)$ & 197 & 250 \\
\hline Ferritin (ug/L) & 24328 & 426806 \\
\hline LDH (U/L) & 400 & 746 \\
\hline CRP (mg/L) & $>400$ & $>230$ \\
\hline ESR (mm/hr) & $>100$ & $>65$ \\
\hline Blood culture & No growth & No growth \\
\hline $\operatorname{ALT}(\mathrm{U} / \mathrm{L})$ & $57 \rightarrow 2266$ & \\
\hline Bilirubin (umol/L) & $9 \rightarrow 94$ & \\
\hline INR & $0.9 \rightarrow 1.64$ & \\
\hline HIV Rapid test & Negative & Negative \\
\hline Hepatitis virus (A, B and C) & Negative & Negative \\
\hline Sputa GXP & Negative & Negative \\
\hline Pericardial fluid & $\begin{array}{c}\text { Exudative, ADA - } \\
40 \text { neutrophil } \\
\text { predominance, } \\
\text { TB-GXP negative, } \\
\text { AFB negative }\end{array}$ & $\begin{array}{c}\text { Exudative, ADA - } \\
\text { 32, neutrophil } \\
\text { predominance, } \\
\text { TB-GXP negative, } \\
\text { AFB negative }\end{array}$ \\
\hline Skin biopsy & & $\begin{array}{l}\text { Inflammatory } \\
\text { exudate consistent } \\
\text { with HSVNVZV }\end{array}$ \\
\hline
\end{tabular}

$\overline{W C C}=$ White Cell Count, $\mathrm{Hb}=$ Haemoglobin, Plt $=$ Platelets, $\mathrm{LDH}=$ Lactose Dehydrogenase, $C R P=C$-Reactive Protein, ESR = Erythrocyte Sedimentation rate $A L T=$ Alanine Aminotransferase, INR = International Normalised Ratio, $A D A=$ Adenosine Deaminase, $A F B=$ Acid Fast Bacilli, HSV = Herpes Simplex Virus, VZV = Varicella Zoster Virus, $T B-G X P=$ Tuberculosis Gene Xpert test. without stranding. The abdominal ultrasonography was normal. The initial diagnostic impression was tuberculous pericarditis with Poncet's disease. A therapeutic and diagnostic pericardiocentesis was performed, and he was started on empiric ATT. A week later, he had ongoing temperature spikes while his rash and arthritis improved. Since he fulfilled the Yamaguchi criteria for AOSD and was not responding to ATT as expected, he was treated with an intravenous steroid pulse of $750 \mathrm{mg}$ over a 3-day period, followed by a remarkable response. He was later started on oral prednisone $30 \mathrm{mg}$ per day and azathioprine 50mg and was discharged a week later for review as an outpatient.

\section{DISCUSSION}

Tuberculosis is a granulomatous disease secondary to Mycobacterium tuberculosis infection. It is a significant public health issue in South Africa - 3\% of the world's tuberculosis cases are from SA and it is the eighth leading cause of death in this country. In 2018, a total of 63000 people died of tuberculosis of which two-thirds were infected with HIV. It is estimated that about 301000 people were diagnosed with tuberculosis in South Africa in 2018. However, according to the 2019 $\mathrm{WHO}$ report, both tuberculosis incidence and mortality rate are decreasing each year.(1)

Pulmonary tuberculosis is the commonest form of presentation. Extra-pulmonary tuberculosis is not uncommon, however, 

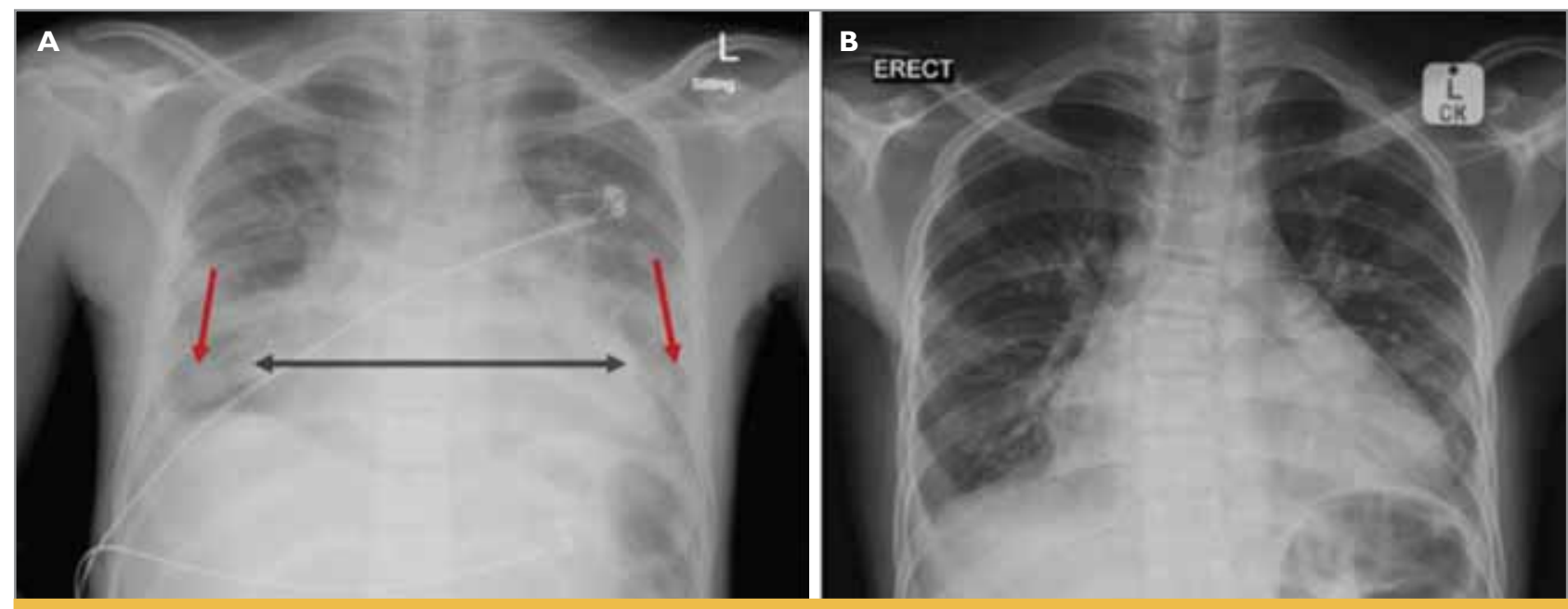

FIGURE 2: Chest radiographs. (A) On admission. A-P film showing a widened mediastinum (black arrow) and lower lung zones infiltrates (red arrow). (B) On recovery. A-P film with resolution of most abnormal features previously described.

TABLE II: Pericardial fluid tests in tuberculous pericarditis. ${ }^{(7)}$

\begin{tabular}{lccc}
\hline Test & Value & Sensitivity & Specificity \\
IFN-gamma & $>$ or $=50 \mathrm{pg} / \mathrm{ml}$ & $92 \%$ & $100 \%$ \\
ADA & $>$ or $=40 \mathrm{U} / \mathrm{l}$ & $87 \%$ & $89 \%$ \\
\hline
\end{tabular}

IFN =Interferon, $A D A=$ Adenosine deaminase.

and more so in immunocompromised patients. Tuberculous pericarditis is not infrequent in $\mathrm{HIV}-\mathrm{I}$ infected patients, and it is the most life-threatening form of extrapulmonary tuberculosis. Tuberculosis causes inflammation of the pericardium leading to pericardial effusion, potentially causing acute circulatory collapse. One of the long-term complications of tuberculous pericarditis is constriction of the pericardium. ${ }^{(2)}$

The treatment for TB pericarditis is the standard ATT consisting of rifampicin, isoniazid, pyrazinamide and ethambutol. Pericardial drainage is also performed in cases of massive pericardial effusion, and subsequently pericardiectomy if constriction develops. Adjunctive therapies to ATT have been evaluated. The Investigation of the Management of Pericarditis (IMPI), a large series of clinical trials that evaluated the effect of prednisolone or Mycobacterium indicus pranii or both, added to the standard ATT regimen, showed no mortality benefit with either of the adjunctive therapies. However, the prednisolone group showed a lower rate of constriction and hospitalisation compared to the placebo group. The increased incidence of malignancy with both interventions, and a marked increase in HIV-related malignancies in the prednisolone group, were matters of concern. ${ }^{(3)}$
Fever is one of the cardinal features of tuberculosis, and cases of disseminated or extrapulmonary forms of tuberculosis are usually associated with high fevers. ${ }^{(4)}$ Tuberculosis-associated fever pattern can be intermittent or recurrent, or present in a double quotidian form, which is 2 temperature spikes per day. ${ }^{(5)}$ SJIA/AOSD are often included in the differential diagnosis of patients presenting with high fevers more than $39^{\circ} \mathrm{C}$, usually as a quotidian (daily) pattern, and often in the afternoon or early evening. ${ }^{(4)}$ Tuberculosis with reactive joint involvement referred to as Poncet's disease can mimic sIIA/AOSD disease due to similar clinical findings. ${ }^{(6)}$ The fever pattern may occasionally assist in differentiating the 2 conditions.

Over and above the clinical features, a serum globulin level of more than $40 \mathrm{~g} / \mathrm{L}$ and a leukocyte count less than $10 \times 10^{\%} / \mathrm{L}$ are highly predictive of tuberculous pericarditis in the appropriate setting. (7) Pericardial fluid investigational variables that can be used for a diagnostic model for tuberculous pericarditis include interferon gamma (IFN gamma) and adenosine deaminase (ADA) (see Table II). The combination of fluid ADA, fluid lymphocyte/neutrophil ratio, HIV status and blood leukocyte count have a diagnostic sensitivity of $96 \%$ and specificity of $97 \%{ }^{(7)}$

SIIA is a systemic autoinflammatory disease mainly affecting the joints of patients below the age of 16 and is responsible for approximately 10\% - 15\% of all cases of JIAs. Similarly, AOSD is a systemic autoinflammatory disease in the population older than 16 years. ${ }^{(8)}$ Multiple diagnostic criteria have been formulated for the diagnosis of AOSD, and among these the most prominent and widely utilised are the Yamaguchi and Fautrel's criteria (Tables III and IV respectively). ${ }^{(8)}$ 
TABLE III: Yamaguchi classification criteria for AOSD. ${ }^{(8)}$

\begin{tabular}{|c|c|c|}
\hline Major criteria & Minor criteria & Exclusion criteria \\
\hline Fever $\geq 39^{\circ} \mathrm{C}$ lasting I week or longer & Sore throat & Infections \\
\hline Arthralgia or arthritis lasting 2 weeks or longer & Recent development of significant lymphadenopathy & Malignancies (mainly malignant lymphoma) \\
\hline Typical rash & Hepatomegaly or splenomegaly & $\begin{array}{l}\text { Other rheumatic disease } \\
\text { (mainly systemic vasculitides) }\end{array}$ \\
\hline \multirow[t]{2}{*}{$\begin{array}{l}\text { Leucocytosis } \geq 10000 / \mathrm{mm}^{3} \text { with } \geq 80 \% \\
\text { polymorphonuclear cells }\end{array}$} & Abnormal liver function tests & \\
\hline & $\begin{array}{l}\text { Negative tests for antinuclear antibody (IF) } \\
\text { and rheumatoid factor (IgM) }\end{array}$ & \\
\hline
\end{tabular}

TABLE IV: Fautrel classification criteria for AOSD..$^{(8)}$

\begin{tabular}{|c|c|}
\hline Major criteria & Minor criteria \\
\hline Spiking fever $\geq 39^{\circ} \mathrm{C}$ & Maculopapular rash \\
\hline Arthralgia & Leukocytosis $\geq 10000 / \mathrm{mm}^{3}$ \\
\hline \multicolumn{2}{|l|}{ Transient erythema } \\
\hline \multicolumn{2}{|l|}{ Pharyngitis } \\
\hline \multicolumn{2}{|l|}{ Polymorphonuclear cells $\geq 80 \%$} \\
\hline \multicolumn{2}{|l|}{ Glycosylated ferritin $\leq 20 \%$} \\
\hline $\begin{array}{l}\text { Four or more major criter } \\
2 \text { minor criteria. }\end{array}$ & ed or 3 major + \\
\hline
\end{tabular}

The combination of a 5-fold increase in serum ferritin and the total glycosylated ferritin of less than $20 \%$ increases the odds for AOSD diagnosis (sensitivity of $43.2 \%$, and specificity of 92.9\%). (9) AOSD-associated massive pericardial effusions resulting in pericardial tamponade have been described in the literature. Therapeutic pericardiocentesis and immunomodulatory therapy have been the cornerstone of therapy. ${ }^{(10)}$

\section{CONCLUSIONS}

Many similarities exist between the clinical presentation of extrapulmonary tuberculosis and that of sJIA/AOSD. While exclusion of an underlying infection is essential in the diagnostic workup of any patient with sJIA/AOSD, the consideration of an auto-inflammatory condition is important in any patient with a presumed diagnosis of tuberculosis. This is true in the absence of an appropriate clinical response to ATT, without microbiological confirmation of tuberculosis.

\section{LEARNING POINTS}

Tuberculosis remains the most common cause of massive effusive pericarditis in South Africa; however, alternative diagnoses must be considered in the appropriate setting.
The quotidian fever pattern and glycosylated ferritin levels below $20 \%$ may assist in the differentiation of tuberculosis in favour of sJIA/AOSD.

1 SIIA/AOSD is associated with markedly elevated ferritin concentration in $70 \%$ of patients. This correlates with disease activity and can be used as a marker for clinical response.

Conflict of interest: none declared.

\section{REFERENCES}

I. Ghebreyesus TA, Kasaeva T, Floyd K, et al. Global tuberculosis report 2019. Geneva: World Health Organisation. WHO/CDS/TB/2019.15

2. Shenje J, Lai RP, Ross IL, et al. Effect of prednisolone on inflammatory markers in pericardial tuberculosis: A pilot study. IJC Heart \& Vasculature 2018; 18:104-108.

3. Mayosi BM, Ntsekhe M, Bosch J, et al. Prednisolone and Mycobacterium indicus pranii in tuberculosis pericarditis. N Engl J Med 2014;37/(I2): $1121-1130$.

4. Saltoglu N, Tasova Y, Midikli D, et al. Fever of unknown origin in Turkey: Evaluation of 87 cases during a nine-year period of study. J Infect 2004; 48(1):81-85

5. Bofinger JJ, Schlossberg D. Fever of unknown origin caused by tuberculosis. Infect Dis Clin North Am 2007;2I (4):947-962.

6. Sood I, Kasundra GM, Supriya PS, et al. A rare case of occult abdominal tuberculosis with Poncet's disease mimicking Adult onset Still's disease. J Midlife Health 2015;6(3): 125- I28.

7. Reuter H, Burgess L, van Vuuren W, et al. Diagnosing tuberculous pericarditis. QJM 2006;99(I2):827-839.

8. Valentin MG, Jamilloux Y, Iwaz J, et al. Adult-onset Still's disease. Autoimmunity Reviews 20 I 4; 13:708-722.

9. Hamidou MA, Denis M, Barbarot S, et al. Usefulness of glycosylated ferritin in atypical presentations of adult onset Still's disease. Ann Rheum Dis 2004; 63:605.

10. Ferreira PC, Silva D, Silva MJ, et al. Adult-onset Still's Disease and cardiac tamponade: A rare association. Tex Heart Inst J 20। 5;42(3):277-280. 